\title{
REGENERAÇÃO NATURAL DO COMPONENTE ARBÓREO E ARBUSTIVO DO PARQUE NATURAL MUNICIPAL DE SANTO ÂNGELO-RS
}

\author{
Alexandre Hüller ${ }^{1}$; Adelita Rauber ${ }^{2}$; Mario Sergio Wolski ${ }^{3}$; Nelson Lopes de Almeida ${ }^{4}$; Silvia \\ Raquel Scherer Wolski ${ }^{5}$
}

(recebido em 30.10.2010 e aceito para publicação em 15.03.2011)

\section{RESUMO}

O conhecimento do comportamento silvicultural das espécies é de extrema importância para o sucesso na implantação de projetos de restauração florestal. Este trabalho tem por objetivo descrever a regeneração natural da área do Parque Natural Municipal de Santo Ângelo - RS (2819'17' S 54ํ15'52" W). Para o estudo utilizou-se parcelas circulares, com $1 \mathrm{~m}$ de raio. Foram inventariados os exemplares arbóreos e arbustivos com altura maior que $30 \mathrm{~cm}$ e DAP $\leq 5 \mathrm{~cm}$. Os parâmetros fitossociológicos avaliados foram: densidade, frequências absoluta e relativa, e altura média. Foram encontrados 78 indivíduos, distribuídos em 26 espécies, pertencentes a 24 gêneros e 14 famílias botânicas. As famílias mais representativas foram Lauraceae, Sapindaceae, Myrtaceae, Fabaceae, Melastomataceae e Solanaceae.

Palavras-chave: fitossociologia, regeneração natural, espécies florestais.

\footnotetext{
1 Biólogo, mestrando em Ciência e Tecnologia de Sementes - UFPEL e Técnico Ambiental da Secretaria Estadual de Meio Ambiente/Departamento de Florestas e Áreas Protegidas - RS, Trav. Honório Lemos, 34, Apartamento 303, Bairro Centro, CEP 98802-350, Santo Ângelo-RS. alexandre-huller@sema.rs.gov.br

${ }^{2}$ Bióloga, Especialista em Ciências Ambientais, Travessa Jose Mendes 317, apartamento 102, Bairro COHAB, CEP 98800-000, Santo Ângelo-RS. adelita_rauber@yahoo.com.br

${ }^{3}$ Geógrafo, M. Sc., Professor Titular do Departamento das Engenharias e Ciência da Computação da Universidade Regional Integrada do Uruguai e das Missões, Campus Santo Ângelo-RS, Rua Universidade das Missões, 464, Bairro Dorneles, CEP 98802-470, Santo Ângelo-RS. mwolski@urisan.tche.br

${ }^{4}$ Engenheiro Florestal, Coordenador Técnico da Associação de Reflorestamento - ARFOM de Santo Ângelo-RS, Rua Sete de Setembro 755, Bairro Centro, CEP 98801-680, Santo Ângelo-RS. nelson.l@terra.com.br

${ }^{5}$ Engenheira Florestal M. Sc., Secretaria da Agricultura, Pecuária, Pesca e Agronegócio do RS, Professora do curso de pós-graduação em Ciências Ambientais, da Universidade Regional Integrada do Uruguai e das Missões, campus Santo Ângelo-RS, Rua Marechal Floriano, 1430, Bairro Centro, CEP 98801-640, Santo Ângelo - RS. srscherer@sol.psi.br.
} 


\section{NATURAL REGENERATION OF TREE AND SHRUB COMPONENT IN SANTO ÂNGELO NATURAL MUNICIPAL PARK, RS}

\section{ABSTRACT}

The knowledge about silvicultural behavior of species is extremely important for a successful implementation of forest restoration projects. This study aims describe natural regeneration in Santo Ângelo Natural Municipal Park area in State of Rio Grande do Sul (28ํ19'17" S $\left.54^{\circ} 15^{\prime} 52^{\prime \prime} \mathrm{W}\right)$. For this research we used circular plots with $1 \mathrm{~m}$ radius. Were inventoried specimens of trees and shrubs taller than $30 \mathrm{~cm}$ and $\mathrm{DBH} \leq 5 \mathrm{~cm}$. The phytosociological parameters were: density, absolute and relative frequency and average height. We found 78 trees belonging to 26 species inside 24 genera and 14 botanical families. The most representative families were: Lauraceae, Sapindaceae, Myrtaceae, Fabaceae, Melastomataceae and Solanaceae.

Keywords: phytosociology, natural regeneration, forest species.

\section{INTRODUÇÃO}

A fragmentação florestal pode interferir na conservação das espécies da fauna e flora local e na distribuição da variabilidade genética entre e dentro das populações. A situação é ainda mais agravada em áreas que se localizam perto de áreas urbanizadas ou em processo de urbanização, devido à pressão antrópica que as cidades exercem sobre estes fragmentos florestais remanescentes (FISZON et al., 2003).

A fragmentação florestal tende a gerar populações reduzidas e isoladas reprodutivamente, com um nível mais elevado de endogamia, o que pode ocasionar depressão endogâmica (FRANKHAM et al., 2002).

A regeneração natural é de extrema importância para a restauração florestal de novas áreas que necessitam de uma recuperação ambiental e também em fragmentos florestais já estabelecidos, pois a mesma auxilia no processo de renovação da composição florística do local e a manutenção das espécies nativas de uma determinada região. 
A expressão regeneração natural é definida como sendo o conjunto de descendentes das plantas arbóreas que se encontram entre $0,1 \mathrm{~m}$ de altura até o limite de diâmetro estabelecido no levantamento estrutural (FINOL, 1971), e consiste ainda em importante indicador de avaliação e monitoramento da restauração de ecossistemas degradados (RODRIGUES e GANDOLFI, 1998).

A forma como a floresta se regenera depende de mecanismos que viabilizem o ingresso e o estabelecimento de novos indivíduos e espécies. Entre estes, a chuva de sementes, banco de sementes do solo, o banco de plântulas e os meios de dispersão de sementes. Por meio da regeneração natural, as florestas apresentam capacidade de se regenerarem de distúrbios naturais ou antrópicos.

A regeneração natural é vital para assegurar que as florestas desempenhem suas funções, pois é a reposição natural das espécies e o surgimento de outras, conforme o nível de desenvolvimento dos estágios seriais, que vão garantir o equilíbrio e a perpetuação dos ecossistemas, (SOUSA JÚNIOR, 2005).

Segundo Seitz (1994), a regeneração natural da vegetação tem recuperado grandes áreas degradadas durante os séculos passados. Afirma também que a regeneração natural da vegetação é o processo mais econômico para recuperar áreas degradadas.

O conhecimento do comportamento silvicultural das espécies é de extrema importância para o sucesso na implantação de projetos de restauração florestal (HÜLLER et al., 2009), para garantir a escolha adequada das espécies, quanto à sua ocorrência, bem como sua capacidade de instalação e desenvolvimento no local.

Portanto, o conhecimento e entendimento dos processos de sucessão ajudam sobremaneira o uso sustentável das florestas, porque diferentes estados de sucessão podem cumprir com diferentes funções e objetivos de manejo (DANIEL e JANKAUSKIS, 1989).

Para a conservação das matas remanescentes e seu manejo adequado, Kageyama (1987) recomenda a adoção de práticas compatíveis com a composição e estrutura destas populações, considerando-se principalmente a forma de ocorrência das espécies, o modo de reprodução e o relacionamento das mesmas com o ambiente, onde todas as interações estão presentes.

Em Santo Ângelo, restam poucos fragmentos florestais localizados próximos à zona urbana, resistindo à pressão antrópica ocasionada pelo crescimento populacional nas áreas urbanas, sendo que muitos já estão necessitando algum tipo de intervenção para sua manutenção ou recuperação. Dentre estes fragmentos, o Parque Natural Municipal de Santo

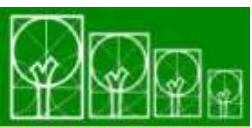

$\mathbf{S} \cdot \mathbf{B} \cdot \mathbf{A} \cdot \mathbf{U}$ Soc. Bras. de Arborização Urbana 
Ângelo se destaca pela área representativa de aproximadamente 13 ha e por sua localização, fazendo parte da mata ciliar do rio ljuí, que é o principal rio da região.

Nesse sentido, este trabalho tem por objetivo descrever a regeneração natural da área do Parque Natural Municipal, para gerar subsídios e somar aos demais estudos que visam à elaboração de um plano de ações e posteriormente um plano de manejo da área do parque.

\section{MATERIAL E MÉTODOS}

A área de estudo localiza-se no Parque Natural Municipal de Santo Ângelo, no município de Santo Ângelo, RS, com coordenadas, 28¹7'56" de latitude Sul e 5491'46" de longitude Oeste. A vegetação da área de estudo faz parte da Floresta Estacional Decidual, abrangendo parte da mata ciliar da bacia do rio ljuí.

O clima na região é subtropical úmido, do tipo Cfa, conforme a classificação de Köppen (MORENO,1961). A temperatura média anual é de $21,8^{\circ} \mathrm{C}$ e a precipitação pluviométrica anual de 1734,5mm, (IRDeR, 2007). O solo predominante é o Latossolo Vermelho Distroférrico Típico (STRECK et al., 2002).

Para o estudo da regeneração natural, utilizou-se parcelas circulares, com raio de $1 \mathrm{~m}$, inserido no centro de dez parcelas de $10 \mathrm{~m}$ de largura por $20 \mathrm{~m}$ de comprimento. Estas parcelas foram usadas para o levantamento fitossociológico da vegetação adulta (HÜLLER, 2009) Figura 1. Em cada uma das dez parcelas, foram inventariados todos os exemplares arbóreos e arbustivos com altura maior que $30 \mathrm{~cm}$ e DAP (Diâmetro a altura do peito)- $\leq$ $5 \mathrm{~cm}$. 


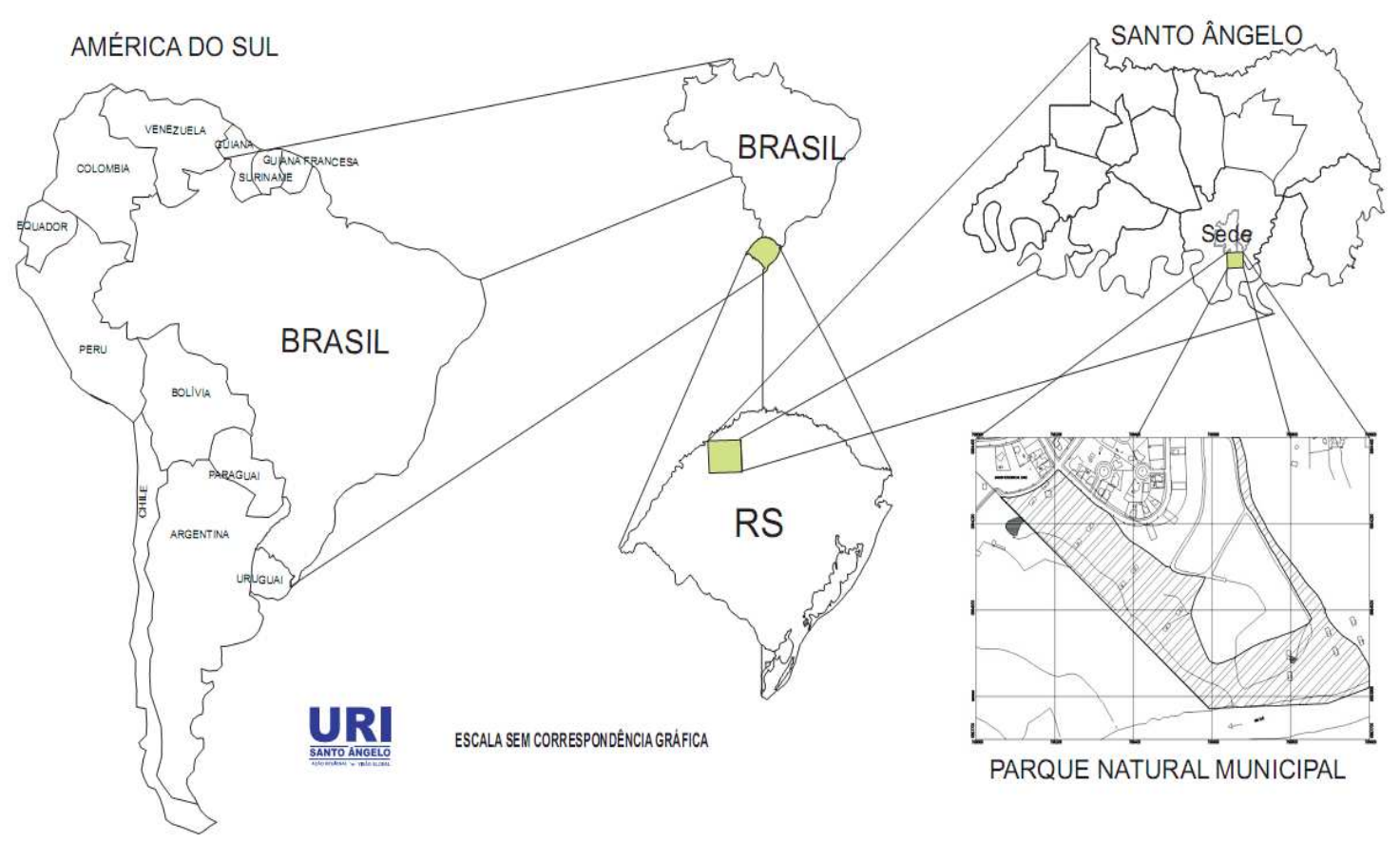

FIGURA 1 - Localização do Parque Natural Municipal de Santo Ângelo, RS.

FIGURE 1 - Localization of Santo Ângelo Natural Municipal Park, RS.

Para a marcação das parcelas utilizou-se a base cartográfica planialtimétrica, com o lançamento das parcelas em faixas, pelo método dos transectos. Para a locação no terreno, foi confeccionada uma planta de locação para cada parcela. As parcelas foram materializadas a campo utilizando equipamentos topográficos (Estação Total) e aplicado o método de coordenadas polares.

Foi estabelecida uma distância de $150 \mathrm{~m}$ entre as parcelas. Os parâmetros fitossociológicos avaliados foram: densidade, frequência absoluta e relativa (BARDDAL et al., 2004), além da altura dos indivíduos. Os dados coletados foram processados com o uso do programa Fitoanálise (CARVALHO JÚNIOR et al., 1999). As espécies foram incluídas nas famílias reconhecidas pelo APG (APG II, 2003). Os levantamentos foram realizados nos meses de maio a outubro do ano de 2008. 


\section{RESULTADOS E DISCUSSÃO}

Nas 10 parcelas avaliadas, foram encontrados 78 indivíduos com DAP $\leq 5 \mathrm{~cm}$, dos quais três estavam mortos. Estes indivíduos estão distribuídos em 26 espécies, pertencentes a 24 gêneros e 14 famílias botânicas (Tabela 1).

A riqueza de espécies é semelhante à descrita por Scherer et al. (2007), que encontraram 23 espécies, pertencentes a 16 famílias botânicas, no Parque Estadual de Itapuã, no município de Viamão - RS, em uma área de mata com $2.900 m^{2}$.

TABELA 1 - Parâmetros fitossociológicos da regeneração natural do Parque Natural Municipal de Santo Ângelo, Santo Ângelo - RS, 2008.

TABLE 1 - Phytosociological parameters of natural regeneration in Santo Ângelo Natural Municipal Park, Santo Ângelo, RS in 2008.

\begin{tabular}{|c|c|c|c|c|c|c|}
\hline Família & Nome científico & $\mathbf{h}$ & DA & DR & FA & FR \\
\hline \multirow[t]{4}{*}{ Lauraceae } & $\begin{array}{l}\text { Cinnamomum amoenum Nees) } \\
\text { Kosterm }\end{array}$ & 2,24 & $1.273,88$ & 1,22 & 10,00 & 1,88 \\
\hline & $\begin{array}{l}\text { Nectandra megapotamica } \\
\text { (Spreng.) Mez }\end{array}$ & 0,69 & $7.643,31$ & 7,31 & 20,00 & 3,77 \\
\hline & Nectandra grandiflora Nees & 0,80 & $1.273,88$ & 1,22 & 10,00 & 1,88 \\
\hline & Ocotea pulchella (Nees) Nees & 1,40 & $1.273,88$ & 1,22 & 10,00 & 1,88 \\
\hline \multirow[t]{4}{*}{ Sapindaceae } & Cupania vernalis Camb. & 0,92 & $11.464,9$ & 10,97 & 60,00 & 11,32 \\
\hline & Allophylus guaraniticus Camb. & 1,05 & $10.191,0$ & 9,75 & 40,00 & 7,54 \\
\hline & Matayba elaeagnoides Radlk. & 0,75 & $2.547,77$ & 2,43 & 20,00 & 3,77 \\
\hline & $\begin{array}{l}\text { Allophylus edulis ((A. St.- } \\
\text { Hilaire) Radlk. }\end{array}$ & 1,47 & $1.273,88$ & 1,22 & 10,00 & 1,88 \\
\hline \multirow[t]{3}{*}{ Myrtaceae } & Eugenia uniflora L. & 1,73 & $7.643,31$ & 7,31 & 40,00 & 7,54 \\
\hline & $\begin{array}{l}\text { Campomanesia xanthocarpa } \\
\text { Berg }\end{array}$ & 1,10 & $3.821,65$ & 3,65 & 20,00 & 3,77 \\
\hline & $\begin{array}{l}\text { Myrcia bombycina (Berg) } \\
\text { Kiaerskou }\end{array}$ & 1,29 & $5.095,54$ & 4,87 & 20,00 & 3,77 \\
\hline \multirow[t]{3}{*}{ Fabaceae } & $\begin{array}{l}\text { Acacia } \\
\text { Grisebach }\end{array}$ & 1,51 & $2.547,77$ & 2,43 & 20,00 & 3,77 \\
\hline & $\begin{array}{l}\text { Dalbergia frutescens (Vell.) } \\
\text { Britt. }\end{array}$ & 1,19 & $5.095,54$ & 4,87 & 20,00 & 3,77 \\
\hline & Calliandra tweediei Bentham & 0,65 & $1.273,88$ & 1,22 & 10,00 & 1,88 \\
\hline \multirow[t]{2}{*}{ Melastomataceae } & $\begin{array}{l}\text { Leandra dasytricha (A.Gray) } \\
\text { Cogn. }\end{array}$ & 1,62 & $1.273,88$ & 1,22 & 10,00 & 1,88 \\
\hline & Miconia cinerascens Miq. & 1,27 & $1.273,88$ & 1,22 & 10,00 & 1,88 \\
\hline \multirow[t]{2}{*}{ Solanaceae } & Cestrum sp. & 1,60 & $3.821,65$ & 3,65 & 30,00 & 5,66 \\
\hline & Solanum mauritianum Scop. & 1,28 & $2.547,77$ & 2,43 & 20,00 & 3,77 \\
\hline Cannabaceae & Celtis tala Gill. & 1,09 & $3.821,65$ & 3,65 & 30,00 & 5,66 \\
\hline Meliaceae & Trichilia elegans A. Jussieu & 1,68 & $6.369,42$ & 6,09 & 30,00 & 5,66 \\
\hline Piperaceae & Piper sp. & 1,41 & $5.095,54$ & 4,87 & 20,00 & 3,77 \\
\hline
\end{tabular}


TABELA 1 - (continuação) Parâmetros fitossociológicos da regeneração natural do Parque Natural Municipal de Santo Ângelo, Santo Ângelo - RS, 2008.

TABLE 1 - (continuation) Phytosociological parameters of natural regeneration in Santo Ângelo Natural Municipal Park, Santo Ângelo, RS in 2008.

\begin{tabular}{llccccc}
\hline \multicolumn{1}{c}{ Família } & \multicolumn{1}{c}{ Nome científico } & h & DA & DR & FA & FR \\
\hline Anacardiaceae & Schinus terebinthifolius Raddi & 0,65 & $6.369,42$ & 6,09 & 20,00 & 3,77 \\
Asteraceae & Baccharis dracunculifolia DC. & 1,80 & $1.273,88$ & 1,22 & 10,00 & 1,88 \\
Apocynaceae & Tabernaemontana catharinensis & 0,63 & $2.547,77$ & 2,43 & 10,00 & 1,88 \\
& DC. & & & & & \\
Euphorbiaceae & Sebastiania brasiliensis Spreng. & 1,65 & $1.273,88$ & 1,22 & 10,00 & 1,88 \\
Rutaceae & Zanthoxylum rhoifolium Lamarck & 0,73 & $2.547,77$ & 2,43 & 10,00 & 1,88 \\
\hline Total & $\mathbf{1 , 2 6}$ & $\mathbf{9 2 . 9 9 3 , 4 5}$ & $\mathbf{1 0 0}$ & $\mathbf{5 3 0}$ & $\mathbf{1 0 0}$ \\
\hline $\mathrm{h}=$ altura das plântulas; DA = densidade absoluta; DR = densidade relativa; FA = frequência absoluta; FR = \\
frequência relativa.
\end{tabular}

As famílias mais representativas foram: Lauraceae e Sapindaceae, com quatro espécies; Myrtaceae, e Fabaceae com três espécies e Melastomataceae e Solanaceae, com duas espécies (Tabela 1).

Cupania vernalis foi a espécie que apresentou os maiores valores fitossociológicos da regeneração natural da área de estudo 10,97 e 11,32 de densidade relativa e frequência relativa, respectivamente. Seguida por Allophylus guaraniticus com, 9,75 e 7,54 densidade e frequência relativa, respectivamente (Tabela 1).

A altura média da regeneração natural dessa área foi de $1,26 \mathrm{~m}$, sendo que a espécie que apresentou a maior altura foi Cinnamomum amoenum com 2,24m, porém com apenas um exemplar. Eugenia uniflora manteve a média de 1,73m de altura, com quatro indivíduos (Tabela 1). Estes dados são semelhantes aos encontrados por Campos e Landgraf (2001), os quais, em estudo da regeneração natural em Furnas - MG obtiveram valores de altura média com 1,43 m e o diâmetro da base foi de $1,25 \mathrm{~cm}$.

Em levantamento fitossociológico do componente arbóreo adulto realizado no mesmo local por Hüller (2009), as espécies mais representativas foram: Sebastiania commersoniana, Luehea divaricata, Diatenopterix sorbifolia e Cupania vernalis, sendo que apenas Cupania vernalis foi encontrada na regeneração natural do presente estudo (Tabela 1). Em comparação do presente estudo com o trabalho de Hüller (2009), das 26 espécies encontradas no estudo da regeneração natural apenas nove coincidiram com o estudo da fitossociologia arbórea adulta (HÜLLER, 2009), através das seguintes espécies: Schinus terebinthifolius, Sebastiania brasiliensis, Nectandra megapotamica, Campomanesia 
xanthocarpa, Eugenia uniflora, Matayba elaeagnoides, Cupania vernalis, Allophylus edulis e Celtis tala. Este resultado mostra uma grande diferença de espécies que ocupam o médio e alto dossel da floresta quando comparadas às espécies que estão se regenerando.

Este fato pode estar relacionado à estrutura sucessional da própria floresta, que em condições de sucessão secundária passa a proporcionar condições mais favoráveis para um banco de sementes das espécies pioneiras e secundárias iniciais e um banco de plântulas para as espécies secundárias tardias e clímax. Harper (1977) comenta que as espécies secundárias e clímax apresentam potencial para formação de bancos de plântulas e de plantas jovens e sobrevivem sob o dossel, podendo morrer se não houver luz para seu desenvolvimento posterior. Além disso, algumas espécies secundárias tardias e climáxicas podem apresentar germinação tardia e assincrônica, principalmente aquelas de tegumento duro e fibroso (FOSTER, 1986).

Medeiros (2004) encontrou em seu trabalho realizado na Amazônia meridional que a similaridade entre a composição florística do banco de sementes e a regeneração natural é baixa e, entre a regeneração natural e indivíduos arbóreos e arbustivos é média a baixa, confirmando os dados obtidos no presente trabalho.

As espécies pioneiras que foram encontradas em pequeno número na regeneração natural, restringindo-se apenas à espécie $S$. terebinthifolius, podem estar sofrendo com a baixa luminosidade, proporcionada pela cobertura das copas das plantas adultas.

Daniel e Jankauskis (1989) confirmam esta hipótese ao encontrarem resultados semelhantes estudando apenas o banco de sementes em floresta de terra firme na Amazônia, onde concluíram que as espécies encontradas nesta condição são do grupo das pioneiras e secundárias iniciais, ambas de pouco valor comercial, mas de grande valor ecológico na reconstrução da floresta.

Apesar disso, os dados apresentados no presente trabalho, em parte são preocupantes, pois segundo HÜLLER (2009), do total de 110 indivíduos amostrados no estudo fitossociológico da mesma floresta, 9,09\% estavam mortos e, se apenas aproximadamente $34 \%$ das espécies foram encontradas também em fase de regeneração, podemos ter neste local, espécies que estão morrendo e não se regenerando, aumentando as chances de extinção dessa espécie nesse fragmento florestal. Pois apesar da grande maioria das espécies que não aparecem no estudo da regeneração natural serem das categorias iniciais, como as pioneiras e secundárias iniciais, o fragmento florestal apresenta sinais de degradação e várias clareiras em seu interior, o que, geralmente apresentaria condições favoráveis também para estas espécies.

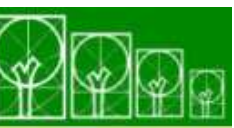

$\mathbf{S} \cdot \mathbf{B} \cdot \mathbf{A} \cdot \mathbf{U}$ Soc. Bras. de Arborização Urbana 
Diante disso, outros fatores devem ser mais bem investigados através de maiores estudos, pois é provável que haja uma soma de fatores que podem estar contribuindo para a baixa riqueza de espécies de determinadas categorias sucessionais neste local, como a ausência de animais frugívoros, por exemplo.

Sugere-se, por fim, a elaboração de um Plano de Manejo do Parque, com a implantação de um plano de recuperação da área, com uma atenção especial para as espécies que apresentaram dificuldade de regeneração neste estudo, especialmente as pioneiras e as secundárias iniciais, aproveitando os conhecimentos apresentados neste trabalho e, ainda motivando novos e mais aprofundados estudos nesta região.

\section{CONCLUSÕES}

As famílias mais representativas na regeneração natural foram Lauraceae, Sapindaceae, Myrtaceae, Fabaceae, Melastomataceae e Solanaceae.

Grande parte das espécies encontradas na regeneração natural são das categorias sucessionais secundárias tardias e clímax.

Apenas 34\% das espécies encontradas na regeneração natural estavam presentes no outro estudo de fitossociologia adulta, com um número reduzido de espécies pioneiras e secundárias iniciais.

\section{REFERÊNCIAS BIBLIOGRÁFICAS}

APG - Angiosperm Phylogeny Group. An update of the angiosperm phylogeny group classification for the orders and families of flowering plants: APG II. Botanical Journal of the Linnean Society, n.141, v.4: p.399-436, 2003.

BARDDAL, M.L. et al. Fitossociologia do sub-bosque de uma floresta ombrófila mista aluvial, no município de Araucária, PR. Ciência Florestal, v.14, n.1,: p.35-45, 2004.

CAMPOS, J.C.; LANDGRAF, P.R.C. Análise da regeneração natural de espécies florestais em matas ciliares de acordo com a distância da margem do lago. Ciência Florestal, v.11, n.2: p.143-151, 2001.

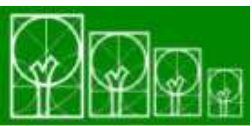

$\mathbf{S} \cdot \mathbf{B} \cdot \mathbf{A} \cdot \mathbf{U}$ Soc. Bras. de Arborização Urbana 
CARVALHO JUNIOR, L.A. et al. Fitoanálise. Versão 4.0. UFSM, Santa Maria, Brasil. Software. 1999.

DANIEL, O; JANKAUSKIS, J. Avaliação de metodologia para o estudo do estoque de sementes do solo, em floresta de terra firme na Amazônia Brasileira. IPEF, 41, p.18-26. 1989.

FINOL, U.H. Nuevos parametros a considerarse en el analisis estrutural de las selvas virgenes tropicales. Revista Florestal Venezolana, n.14, v.21, p.29-42, 1971.

FISZON, J.T. et al. Fragmentação de Ecossistemas: Causas, efeitos sobre a biodiversidade e recomendações de políticas públicas. MMA/SBF, Brasília, Brasil, p.6599. 2003.

FOSTER, S. A. On adaptative value of large seeds for tropical moist forest trees: a review and synthesis. Biol. Rev., v.52, p. 260-269, 1986.

FRANKHAM, R.; BALLOU, J. D.; BRIDGE, D. A. Introduction to Conservation Genetics. Cambridge, UK: Cambridge University Press, 617 p. 2002.

HARPER, J.L. Population biology of plants. London: Academic Press, 1977. 892p.

HÜLLER, A. Estrutura fitossociológica da vegetação arbórea do Parque Natural Municipal de Santo Ângelo, RS. Monografia, Universidade Regional Integrada, Brasil, 11p. 2009.

HÜLLER, A. et al. A comparative study of four tree species used in riparian forest restoration along Uruguay River, Brazil. Revista Árvore, v.33, n.2, p.297-304. 2009.

IRDeR - Instituto Regional de Desenvolvimento Rural. Estação Pluviométrica. FIDENE/UNIJUÍ, Augusto Pestana, Brasil, s/paginação. 2007. 
KAGEYAMA, P.Y. Conservação "in situ" dos recursos genéticos de plantas. Série IPEF, n. 35, v.1, p.7-40. 1987.

MEDEIROS, R.A. Dinâmica de sucessão secundária em floresta de transição na Amazônia meridional. Dissertação de Mestrado, Cuiabá, Brasil, 104p. 2004.

MORENO, J.A. Clima do Rio Grande do Sul. Secretaria da Agricultura do Rio Grande do Sul, Porto Alegre, Brasil, 73p. 1961.

RODRIGUES, R.R.; GANDOLFI, S. Restauração de florestas tropicais: subsídios para uma definição metodológica e indicadores de avaliação e monitoramento. In: DIAS, L. E.; MELO, J. W. V. CONGRESSO BRASILEIRO SOBRE RECUPERAÇÃO DE ÁREAS DEGRADADAS. Viçosa. 1998. Anais... SOBRADE / UFV, 1998. p. 203-215.

SCHERER, A.; MARASCHIN-SILVA, F.; BAPTISTA, L. R. de M. Regeneração arbórea num capão de restinga no Rio Grande do Sul, Brasil. Iheringia, Série Botânica, v. 62, n.1-2, p.89-92. 2007.

SEITZ, R. A. A regeneração natural na recuperação de áreas degradadas. In: SIMPÓSIO SULAMERICANO,1.; SIMPÓSIO NACIONAL DE RECUPERAÇÃO DE ÁREAS DEGRADADAS, 2., 1994. Foz do Iguaçu. Anais... Foz do Iguaçu: FUPEF, 1994. p. 103-110.

SOUSA JÚNIOR, G.A. Zoneamento da faixa tampão do reservatório da UHE-Camargos e avaliação de sua regeneração natural. Dissertação de Mestrado, Lavras, Brasil,103p. 2005.

STRECK, E.V et al. Solos do Rio Grande do Sul. Universidade Federal do Rio Grande do Sul, Porto Alegre, Brasil, 107p. 2002.

UHL, C.; CLARK, K.; MAQUIRINO, P. Vegetation dynamics in Amazonian treefall gaps. Ecology., v.69, p.751-763. 1988. 